\title{
Olivopontocerebellar Atrophy
}

National Institute of Neurological Disorders and Stroke (NINDS)

\section{Definitions}

Multiple System Atrophy

Defined by National Institute of Neurological Disorders and Stroke (NINDS)

\section{Source}

National Institute of Neurological Disorders and Stroke (NINDS). Olivopontocerebellar Atrophy Information Page.

Olivopontocerebellar atrophy (OPCA) is a term that describes the degeneration of neurons in specific areas of the brain - the cerebellum, pons, and inferior olives. OPCA is present in several neurodegenerative syndromes, including inherited and non-inherited forms of ataxia (such as the hereditary spinocerebellar ataxia known as Machado-Joseph disease) and multiple system atrophy (MSA), with which it is primarily associated: Multiple System Atrophy

OPCA may also be found in the brains of individuals with prion disorders and inherited metabolic diseases. The characteristic areas of brain damage that indicate OPCA can be seen by imaging the brain using CT scans or MRI studies. 